\title{
Limitations of Small-Signal Modeling of Grid-Connected VSCs under Phase Jumps and Frequency Deviations
}

\author{
Hongyang Zhang, Member, IEEE, Xiongfei Wang, Senior Member, IEEE, Stefan Östlund, Senior Member, IEEE,
}

\begin{abstract}
The stability of grid-connected voltage source converters (VSCs) is commonly studied by the small-signal model in the synchronous reference $(d q)$ frame. With phase-locked loop dynamic from VSCs, it is inevitable to convert the small-signal model to a common $d q$ frame. When linearizing the frame transformation, the trigonometric functions of the angle are approximated by first-order terms. In this letter, the limitation of such linearization on the $d q$-transformation is discussed. The accuracy of the model, in presence of phase jumps and frequency variations, are examined. It is found that even under small phase jumps, mismatches are found between the small-signal model (SSM) and a nonlinear model (NM). It is further revealed that during the dynamics of frequency variation, significant mismatches can occur between the SSM and the NM.
\end{abstract}

Index Terms-Synchronous reference $(d q)$ frame, frame transformation, voltage source converters (VSCs), small-signal model (SSM), nonlinear model (NM)

\section{INTRODUCTION}

$\mathbf{S}$ TABILITY of a three-phase grid-connected voltage source converter (VSC) configuration is often analyzed by using a synchronous reference $(d q)$-frame small-signal model. In the linearization process, the nonlinearities in the trigonometric functions of $d q$ transformation and also within the power calculations are approximated by first-order functions around a steady-state operating point [1].

The $d q$-frame small-signal modeling usually considers that the VSC is connected to a stiff source with fixed amplitude and frequency [1], [2]. However, to model the power system disturbances such as phase jumps and frequency variations, the phase of the source voltage would also change. Phase jumps are normal power system phenomena which can have many causes [3]. Moreover, in a VSC dominated grid, occurrence of frequency deviations tends to be more frequent, than in the conventional grid governed by synchronous generators [4].

In this letter, the limitations of the $d q$-frame small-signal modeling are revealed in the presence of phase jumps and frequency variations. The small-signal models are constructed by state-space modeling and two sets of reference systems are used to respectively reflect the issues under phase jumps and frequency variations. In [5], the accuracy problems of the $d q$-frame small-signal model, in presence of phase jump is discussed. It is stated that the model accuracy is preserved when the phase variation is below $7^{\circ}$. However, as shown in this letter the inaccuracy of the small-signal model always exists under phase-jump events, regardless of the size of phase jump. Moreover, it is found that the small-signal model is no longer accurate when the system frequency deviates. To effectively evaluate the system stability, the small-signal model should be adapted to a particular system frequency. Numerical simulations confirm the theoretical findings.

\section{SMall-Signal Modeling}

In this letter, the vectors that represent current and voltage variables are expressed, respectively, by $i$ and $u$. For instance, the source voltage vector is expressed as $u_{s}^{s}$, where the superscript ' $s$ ' indicates that the variable is on the $\alpha \beta$ frame. On the other hand, the $d q$-frame variables are denoted without the superscript ' $s$ ' . As shown in [1], the dynamic impact of the phase-locked loop (PLL) introduces a so-called converter $d q$ frame. In the converter $d q$ frame, a superscript ' $c$ ' is added for distinction from the grid $d q$ frame (which is the $d q$ frame with no PLL impact), e.g., $u_{s}^{c}$ is on the converter $d q$ frame and $u_{s}$ is on the grid $d q$ frame. The transformation from $\alpha \beta$ frame to $d q$ frame yields

$$
\begin{aligned}
u_{s}^{c} & =e^{-j(\omega t+\theta)} u_{s}^{s}=e^{-j(\omega t+\theta)} u_{s} e^{j\left(\omega_{s} t+\theta_{s}\right)} \\
& =u_{s} e^{-j\left[\left(\omega-\omega_{s}\right) t+\left(\theta-\theta_{s}\right)\right]}
\end{aligned}
$$

where $\omega$ and $\theta$ are the angular velocity and angle of the PLL respectively. $\omega_{s}$ and $\theta_{s}$ are respectively the nominal angular velocity and the angle of $u_{s}^{s}$. From (1), we note that when $\omega=\omega_{s}=\omega_{1}\left(\omega_{1}=2 \pi f_{1}\right.$, where $f_{1}$ is the nominal system frequency), (1) is reduced as ${ }^{2}$

$$
u_{s}^{c}=u_{s} e^{-j\left(\theta-\theta_{s}\right)}=u_{s} e^{-j \Delta \theta}
$$

where $\Delta \theta$ is the angle difference between $u_{s}^{c}$ and $u_{s}$. For a small $\Delta \theta, \cos \Delta \theta \approx 1, \sin \Delta \theta \approx \theta$, (2) can be linearized as

$$
u_{s}^{c}=u_{s}(1-j \Delta \theta) .
$$

Expanding (3) yields

$$
u_{\mathrm{sd}}^{c}=u_{\mathrm{sd}}+\Delta \theta u_{\mathrm{sq}} \quad u_{\mathrm{sq}}^{c}=u_{\mathrm{sq}}-\Delta \theta u_{\mathrm{sd}} .
$$

Further linearizing (4) yields

$$
\Delta u_{\mathrm{sd}}^{c}=\Delta u_{\mathrm{sd}}+u_{\mathrm{sq} 0} \Delta \theta \quad \Delta u_{\mathrm{sq}}^{c}=\Delta u_{\mathrm{sq}}-u_{\mathrm{sd} 0} \Delta \theta .
$$

\footnotetext{
${ }^{1}$ In this letter, the subscript ' $s$ ', 'pcc', 'c' stand for 'source', 'points of common coupling', 'converter', respectively.

${ }^{2}$ To represent perturbation variables, a prefix $\Delta$ is used, e.g., $\Delta u_{s}$. A constant representing a steady-state operating point contains a ' 0 ' in the end of its subscript, e.g., $u_{\mathrm{s} 0}$.
} 


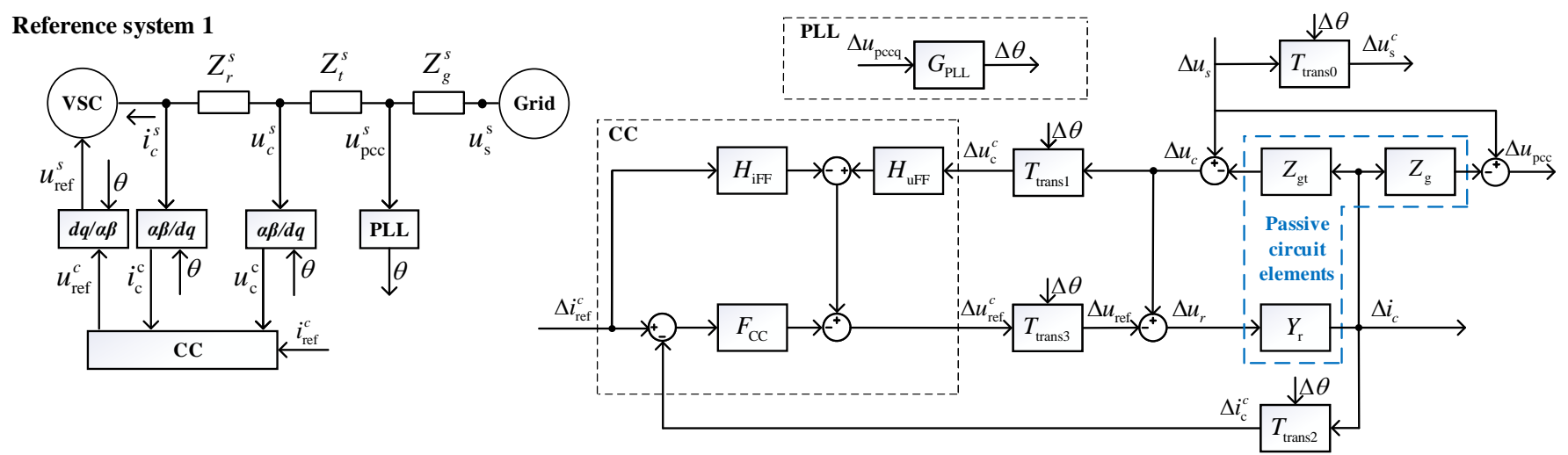

(a)

(b)

Fig. 1. Reference system 1. (a) A grid-following VSC connected to a stiff source. (b) The small-signal model of (a).

\section{A. Reference System 1 with Phase Jump}

Reference system 1 is shown in Fig. 1(a) and the layout of its linearized system is illustrated in Fig. 1(b). As shown in Fig. 1(a), a grid-following VSC is connected to a source via the phase reactor impedance $Z_{r}^{s}$, the transformer impedance $Z_{t}^{s}$, and the grid impedance $Z_{g}^{s}$. To facilitate the analysis, the VSC dc-bus dynamics are not modeled by assuming that an infinite energy source is connected on the dc side. The $\mathrm{CC}$ and PLL are considered. The CC contains the PI regulator $\left(F_{\mathrm{CC}}\right)$, current-reference feedforward filter $\left(H_{\mathrm{iFF}}\right)$, and the converterbus voltage feedforward filter $\left(H_{\mathrm{uFF}}\right)$ [2].

$$
\begin{gathered}
F_{\mathrm{CC}}=\left[\begin{array}{cc}
k_{\mathrm{pCC}}\left(1+\frac{k_{\mathrm{iCC}}}{s}\right) & 0 \\
0 & k_{\mathrm{pCC}}\left(1+\frac{k_{\mathrm{iCC}}}{s}\right)
\end{array}\right] \\
H_{\mathrm{iFF}}=\left[\begin{array}{cc}
\frac{s}{1+s \tau_{\mathrm{iFF}}} & -\omega_{1} \\
\omega_{1} & \frac{s}{1+s \tau_{\mathrm{iFF}}}
\end{array}\right] L_{r} \quad H_{\mathrm{uFF}}=\left[\begin{array}{cc}
\frac{1}{1+s \tau_{\mathrm{uFF}}} & 0 \\
0 & \frac{1}{1+s \tau_{\mathrm{uFF}}}
\end{array}\right]
\end{gathered}
$$

where $\tau_{\mathrm{iFF}}, \tau_{\mathrm{uFF}}$ are the filter time constants, $L_{r}$ is the phase reactor inductance in p.u.

The linearized closed-loop transfer function of the PLL can be derived as [1]

$$
\Delta \theta=\underbrace{\frac{h_{\mathrm{PLL}} f_{\mathrm{PLL}}}{s+h_{\mathrm{PLL}} f_{\mathrm{PLL}}}}_{G_{\mathrm{PLL}}} \Delta u_{\mathrm{pccq}}
$$

where $h_{\mathrm{PLL}}=\frac{1}{1+s \tau_{\mathrm{PLL}}}$ and $f_{\mathrm{PLL}}=k_{\mathrm{pPLL}}\left(1+\frac{k_{\mathrm{PLL}}}{s}\right)$. In Fig. 1 (b), the frame-transformation dynamics between the variables in the two $d q$ frames are modeled by the transfer matrices $T_{\text {trans0 } 0}, T_{\text {trans1 }}, T_{\text {trans2 }}$, and $T_{\text {trans3 } 3}$. For instance, the inputs for $T_{\text {trans0 }}$ are: $\Delta u_{\mathrm{sd}}, \Delta u_{\mathrm{sq}}$, and $\Delta \theta$ and its outputs are: $\Delta u_{\mathrm{sd}}^{c}$, $\Delta u_{\mathrm{sq}}^{c}[2]$

$$
\begin{gathered}
T_{\text {trans } 0}=\left[\begin{array}{ccc}
1 & 0 & u_{\mathrm{sq} 0} \\
0 & 1 & -u_{\mathrm{sd} 0}
\end{array}\right] \quad T_{\text {trans } 1}=\left[\begin{array}{ccc}
1 & 0 & u_{\mathrm{cq} 0} \\
0 & 1 & -u_{\mathrm{cd} 0}
\end{array}\right] \\
T_{\text {trans } 2}=\left[\begin{array}{ccc}
1 & 0 & i_{\mathrm{cq} 0} \\
0 & 1 & -i_{\mathrm{cd} 0}
\end{array}\right] \quad T_{\text {trans } 3}=\left[\begin{array}{ccc}
1 & 0 & -u_{\text {refq } 0} \\
0 & 1 & u_{\text {refd } 0}
\end{array}\right] .
\end{gathered}
$$

The passive circuit components are expressed as

$$
\begin{aligned}
Y_{r}=Z_{r}^{-1} & =\left[\begin{array}{cc}
s & -\omega_{1} \\
\omega_{1} & s
\end{array}\right] L_{r} \quad Z_{g}=\left[\begin{array}{cc}
s & -\omega_{1} \\
\omega_{1} & s
\end{array}\right] L_{g} \\
Z_{\mathrm{gt}} & =\left[\begin{array}{cc}
s & -\omega_{1} \\
\omega_{1} & s
\end{array}\right] L_{\mathrm{gt} .}
\end{aligned}
$$

where $Y_{r}$ is the phase-reactor admittance matrix, $L_{g}$ represents the grid inductance in p.u., $L_{\mathrm{gt}}$ refers to the sum of the grid inductance and transformer inductance in p.u.

The closed-loop dynamics of the system in Fig. 1(b) can be summarized as the reference tracking $\left(\Delta i_{\mathrm{c}}^{c} \rightarrow \Delta i_{\text {ref }}^{c}\right)$ and disturbance rejection $\left(\Delta i_{\mathrm{c}}^{c} \rightarrow 0\right)$ transfer matrices:

$$
\Delta i_{c}^{c}=T_{\mathrm{iU}} \Delta i_{\mathrm{ref}}^{c}+T_{\mathrm{iDU}} \Delta u_{\mathrm{s}}
$$

where $T_{\mathrm{iU}}$ and $T_{\mathrm{iDU}}$ are the corresponding closed-loop transfer matrices.

\section{B. Reference System 2 with Frequency Deviation}

To study the impact of system frequency deviation, reference system 2 is created, see Fig. 2(a). Its corresponding smallsignal model is constructed and shown in Fig. 2(b). In contrast to the reference system 1 , the swing dynamic of synchronous generator $(\mathrm{SG})$ is included in the source frequency, and the outer power-control loop dynamics of the VSC are considered. Consequently, the whole system consists of two parts: the first part represents the swing equations and frequency-regulation dynamics from the governor and turbine of the SG; the second part models the closed-loop dynamics of the grid-following converter. The two parts of the models are integrated by $u_{s}$ on the grid $d q$ frame. In the first part of the model, the governor and turbine dynamics [6], as well as swing dynamics are modeled as

$$
\begin{aligned}
& H_{\mathrm{gov}}= \frac{k_{\mathrm{pfs}}}{1+s T_{G}} \quad H_{\text {turbine }}=\frac{1+s F_{\mathrm{HP}} T_{\mathrm{RH}}}{\left(1+s T_{\mathrm{CH}}\right)\left(1+s T_{\mathrm{RH}}\right)} \\
& \Delta \dot{\theta}_{s}=\omega_{1} \Delta \omega_{s} \\
& \Delta \dot{\omega}_{s}=\frac{1}{M}\left[\Delta p_{\mathrm{gov}}+\Delta p_{m}-(\underbrace{\Delta p_{L}+\Delta p_{f}}_{\Delta p_{e}})\right]
\end{aligned}
$$




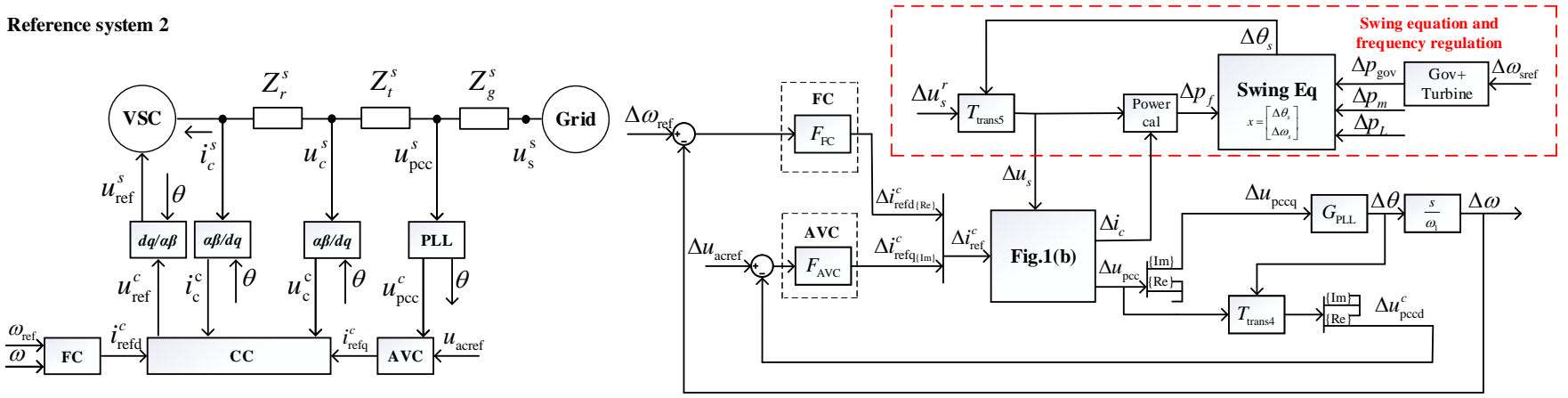

(a)

(b)

Fig. 2. Reference system 2. (a) A grid-following VSC connected to a source with swing dynamic. (b) The small-signal model of (a).

where $k_{\mathrm{pfs}}$ is the droop gain for the governor, $T_{G}, F_{\mathrm{HP}}, T_{\mathrm{RH}}$, $T_{\mathrm{CH}}$, and $T_{\mathrm{RH}}$ are the filter time constants, $\Delta \theta_{s}$ and $\Delta \omega_{s}$ are the rotor angle and angular velocity deviations respectively. $\Delta p_{\text {gov }}, \Delta p_{m}, \Delta p_{e}, \Delta p_{L}$, and $\Delta p_{f}$ are the deviation variables for the governor power, mechanical power, electrical power, load power, and converter output power respectively. $\Delta p_{\text {gov }}=$ $H_{\text {gov }} H_{\text {turbine }} \Delta \omega_{\text {sref }}, \Delta \omega_{\text {sref }}$ is the reference angular velocity for the governor. $\Delta p_{f}=u_{\mathrm{sd} 0} \Delta i_{\mathrm{cd}}+u_{\mathrm{sq} 0} \Delta i_{\mathrm{cq}}+i_{\mathrm{cd} 0} \Delta u_{\mathrm{sd}}+i_{\mathrm{cq} 0} \Delta u_{\mathrm{sq}}$. $\Delta p_{m}$ and $\Delta p_{L}$ are treated as external inputs.

An inverse $d q$ transformation is applied on $\Delta u_{\mathrm{s}}^{r}$, i.e., a frame transformation from the rotor $d q$ frame (with superscript ' $r$ ') to the grid $d q$ frame. A transfer function matrix $T_{\text {trans5 }}$ is applied for a transformation of inputs $\Delta u_{\mathrm{sd}}^{r}, \Delta u_{\mathrm{sq}}^{r}$, and $\Delta \theta_{s}$ to the outputs $\Delta u_{\mathrm{sd}}$ and $\Delta u_{\mathrm{sq}}$. To regulate the voltage $\left|u_{\mathrm{pcc}}\right|$ and angular velocity $\omega_{\mathrm{pcc}}$ at the PCC, the ac-busvoltage controller (AVC) and frequency controller (FC) are implemented to generate $i_{\mathrm{ref}}^{c}$. $\omega_{\mathrm{pcc}}$ is estimated by the PLL $\left(\omega=\omega_{1}+\Delta \omega=\omega_{1}+\dot{\Delta \theta} / \omega_{1}\right)$ and $\omega$ is regulated to $\omega_{\text {ref }}$ (see Fig. 2). The AVC regulator $F_{\mathrm{AVC}}$ contains only the integral controller. $T_{\text {trans } 4}$ represents the frame transformation dynamics from $\Delta u_{\mathrm{pcc}}$ to $\Delta u_{\mathrm{pcc}}^{c}$. The FC consists of proportional (droop) and derivative (virtual inertia) controllers $F_{\mathrm{FC}}$

$$
\begin{gathered}
F_{\mathrm{AVC}}=\frac{k_{\mathrm{iAVC}}}{s} \quad F_{\mathrm{FC}}=-k_{\mathrm{pf}}-k_{\mathrm{pfd}} s \\
T_{\text {trans4 }}=\left[\begin{array}{ccc}
1 & 0 & u_{\mathrm{pccq} 0} \\
0 & 1 & -u_{\mathrm{pccd} 0}
\end{array}\right] \quad T_{\text {trans } 5}=\left[\begin{array}{ccc}
1 & 0 & -u_{\mathrm{sq} 0} \\
0 & 1 & u_{\mathrm{sd} 0}
\end{array}\right] .
\end{gathered}
$$

where $k_{\mathrm{iAVC}}$ is the integral gain of the AVC, $k_{\mathrm{pf}}$ and $k_{\mathrm{pfd}}$ are the proportional and derivative gain of the FC respectively.

\section{Limitations of Small-Signal Models}

\section{A. Phase Jumps}

The purpose of reference system 1 is to check the accuracy of the small-signal model when a phase jump $\Delta \theta_{s}$ occurs at the source. To represent $\Delta \theta_{s}$ in terms of $\Delta u_{\mathrm{s}}$, we have $\Delta u_{\mathrm{sd}}=\cos \left(\Delta \theta_{s}\right)-u_{\mathrm{sd} 0}, \Delta u_{\mathrm{sq}}=\sin \left(\Delta \theta_{s}\right)$.

With $i_{\mathrm{c} 0}=j 0.5$ p.u., $L_{g}=0.1$ p.u., and $u_{\mathrm{s} 0}=1$ p.u., Fig. 3 (a)-(d) respectively show the response of $i_{\mathrm{cd}}^{c}, i_{\mathrm{cq}}^{c}, u_{\mathrm{sd}}^{c}$, and $u_{\mathrm{sq}}^{c}$ under $\Delta \theta_{s}=0.0628 \mathrm{rad}$. It is straightforward to analyze the response of $\Delta u_{\mathrm{s}}^{c}$ since its dynamic is only impacted by the size of phase jump in the system $\left(\Delta u_{\mathrm{s}}=-0.002+j 0.0628\right.$ p.u.) and the PLL dynamics from $\Delta \theta$. In Fig. 3 (a) and (b), it can be observed that after the phase jump, the CC regulates the current at $i_{\text {ref }}=j 0.5$ p.u. A close matching between the nonlinear model (NM) (solid curves) and the small-signal model (SSM) (dashed curves) is observed for the response of $i_{\mathrm{c}}^{c}$. However, a difference between the models is found in Fig. 3(c) where $\Delta u_{\mathrm{sd}}^{c}=-0.002$ p.u. in the SSM while $u_{\mathrm{sd}}^{c}$ is restored after the disturbance in the NM. This mismatch can be intuitively checked by $T_{\text {trans0 }}$ in (9): with $u_{\text {sq0 } 0}=0$ p.u., $\Delta u_{\mathrm{sd}}^{c}=\Delta u_{\mathrm{sd}}=-0.002$ p.u. The PLL dynamics on $\Delta u_{\mathrm{sd}}^{c}$ are thus overlooked in the SSM compared to the NM. On the other hand, the PLL dynamic on $\Delta u_{\mathrm{sq}}^{c}$ is correctly modeled in the SSM since $u_{\mathrm{sd} 0}=1$ p.u. The limitation for the firstorder approximation of the trigonometric functions in (9) can be proved by performing Maclaurin series expansion of the trigonometric functions up to their second-order terms:

$$
\begin{aligned}
& \Delta u_{\mathrm{sd}}^{c}=\Delta u_{\mathrm{sd}}+u_{\mathrm{sq} 0} \Delta \theta-u_{\mathrm{sd} 0} \frac{\Delta \theta^{2}}{2}+\Delta u_{\mathrm{sq}} \Delta \theta \\
& \Delta u_{\mathrm{sq}}^{c}=\Delta u_{\mathrm{sq}}-u_{\mathrm{sd} 0} \Delta \theta-u_{\mathrm{sq} 0} \frac{\Delta \theta^{2}}{2}-\Delta u_{\mathrm{sd}} \Delta \theta .
\end{aligned}
$$

Using (18) and (19), where the $\Delta \theta$ is the outputs of the SSM, the time-domain response (dotted curves) is overlapped with the NM, see Fig. 3(c) and (d) ${ }^{3}$. This fact shows that the existing small-signal model fails to accurately model VSC dynamics during phase jump events, even when the phase jump is smaller than $7^{\circ}\left(0.0628 \mathrm{rad} \approx 3.6^{\circ}\right)$. In [5], it is discussed to use a nonlinear model for the frame transformation by considering the first three terms of (18) and (19). Yet overlooking relevant second-order terms $\Delta u_{\mathrm{sq}} \Delta \theta$ and $\Delta u_{\mathrm{sd}} \Delta \theta$ in (18) and (19) would not match the response to the NM, see the dashdotted curves in Fig. 3(c) and (d).

\section{B. System Frequency Deviations}

A step $\Delta p_{L}=0.1$ p.u. is applied in the reference system 2, the response of $\omega_{s}, \omega$, and $\theta$ are shown in Fig. 4 (a), (b), and (c) respectively. The time-domain simulations match between

\footnotetext{
${ }^{3} \mathrm{~A}$ model having second-order terms is already not a small-signal model. Thus, calculating of (18) and (19) is just for verification purpose.
} 

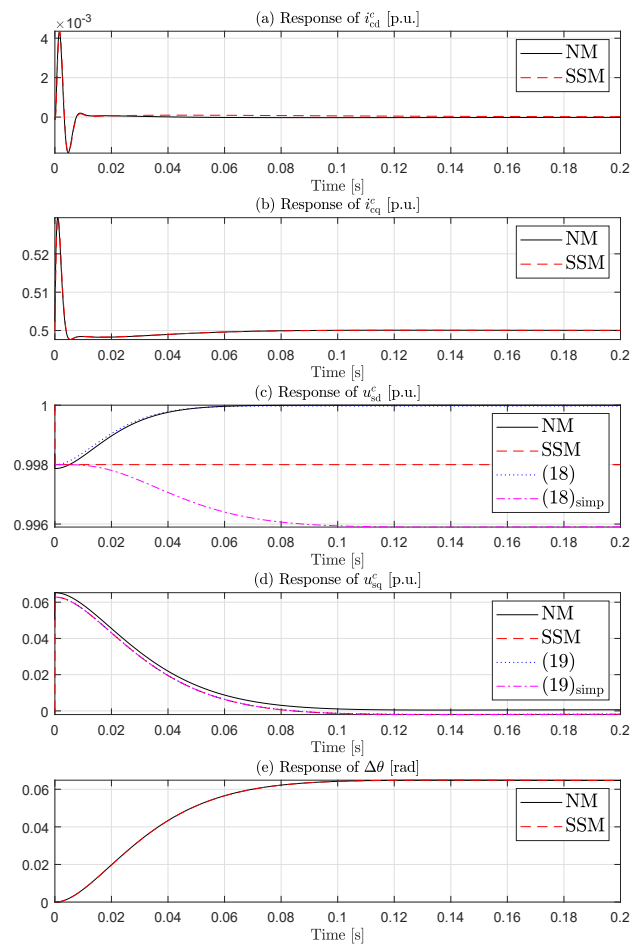

Fig. 3. $\Delta \theta_{s}=0.0628$ rad. (a) Response of $i_{\mathrm{cd}}^{c}$ in the NM and SSM, (b) Response of $i_{\mathrm{cq}}^{c}$ in the NM and SSM, (c) Response of $u_{\mathrm{sd}}^{c}$ in the NM, SSM, calculation of (18), and calculation of (18) with first three terms, (d) Response of $u_{\mathrm{sq}}^{c}$ in the NM, SSM, calculation of (19), and calculation of (19) with first three terms, (e) Response of $\Delta \theta$ in the NM and SSM.

the NM and SSM. The steady-state frequency deviation yields -0.004 p.u., with $k_{\text {pfs }}=20$ p.u. and $k_{\text {pf }}=5$ p.u. However, with even a small deviation of $\Delta \omega$ from 0 p.u., $\Delta \theta$ keeps growing [see Fig. 4(c)] since $\Delta \theta=\omega_{1} \int \Delta \omega d t$.

With the frequency deviation, the large $\Delta \theta$ creates fundamental problems for the $d q$ frame small-signal modeling since $\omega \neq \omega_{1}$ in (1) and thus the linearization in (2) can not be achieved. The response of $i_{\mathrm{cd}}^{c}$ and $i_{\mathrm{cq}}^{c}$ are respectively shown in Fig. 4(d) and (e) for the NM and SSM. Good accuracy is found for the dynamic of $i_{\mathrm{cd}}^{c}$, however, not for $i_{\mathrm{cq}}^{c}$. This significant mismatch on the $d q$ frame current can be explained by the limitation of small-signal modeling. With $u_{\text {acref }}=1.05$ p.u., $u_{\mathrm{s} 0}=1.0$ p.u., and $L_{\mathrm{g}}=0.1$ p.u., we have $i_{\mathrm{c} 0}=j 0.5$ p.u. It is noticed that at Time $=6 \mathrm{~s}, \Delta \theta=-10 \mathrm{rad}$ in Fig. 4(c). According to the input-output relations of $T_{\text {trans } 2}$ in (10), $\Delta i_{\mathrm{cq}}^{c}=\Delta i_{\mathrm{cq}}$ and $\Delta i_{\mathrm{cd}}^{c}$ deviates as large as 5 p.u. from $\Delta i_{\mathrm{cd}}$ due to $\Delta \theta=-10 \mathrm{rad}$, see Fig. 4(f). To summarize, when $\Delta \omega \neq 0$ in the steady state, $\Delta \theta$ does not settle to a constant value, the time-domain response of the SSM thus does not match the NM since a new steady-state solution of the SSM can not be achieved. This also implies that when the system frequency deviates, the small-signal stability analysis of VSC should always be evaluated at a steady-state frequency $f_{0}$.

\section{CONCLUSIONS}

In this letter, the limitations of the small-signal model of grid-connected VSCs is discussed in the presence of phase jumps and frequency variations. Inaccurate dynamics of the
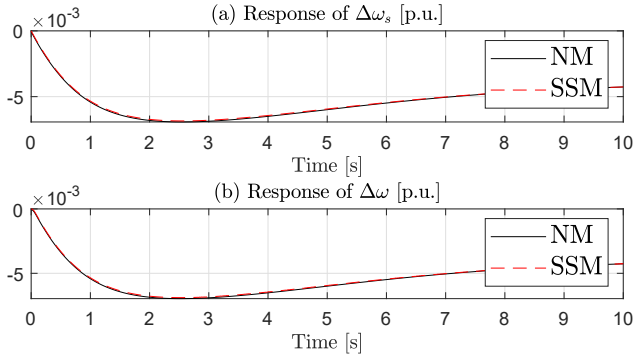

(c) Response of $\Delta \theta[\mathrm{rad}]$

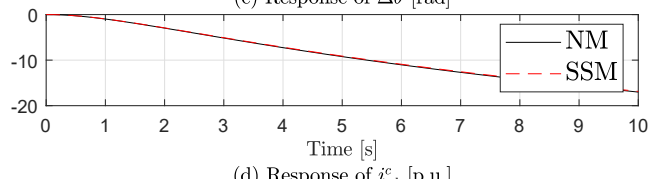

(d) Response of $i_{\mathrm{cd}}^{c}$ [p.u.]

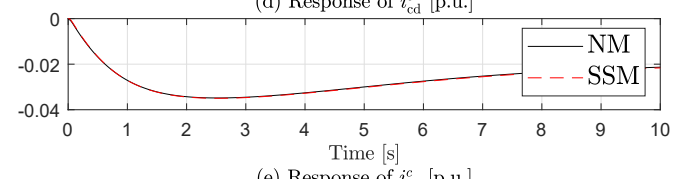

(e) Response of $i_{\mathrm{cq}}^{c}$ [p.u.]

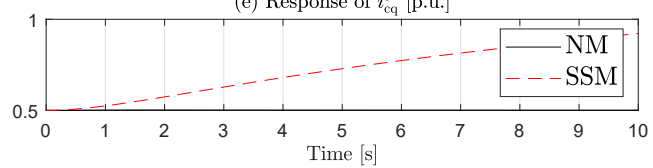

(f) Response of $i_{\mathrm{cd}}$ and $i_{\mathrm{cd}}^{c}$ in the SSM [p.u.]

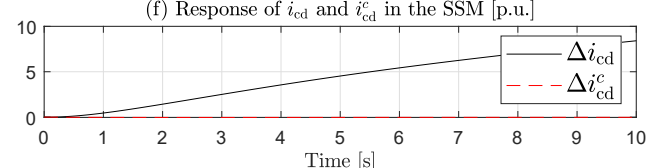

(g) Response of $i_{\mathrm{cq}}$ and $i_{\mathrm{cq}}^{c}$ in the SSM [p.u.]

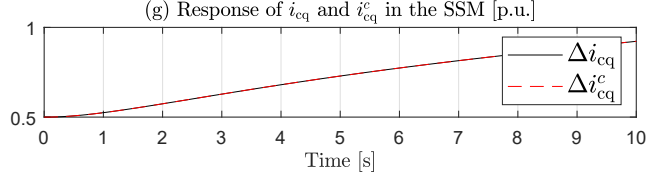

Fig. 4. (a) Response of $\Delta \omega_{s}$ in the NM and SSM, (b) Response of $\Delta \omega$ in the NM and SSM, (c) Response of $\Delta \theta$ in the NM and SSM, (d) Response of $i_{\mathrm{cd}}^{c}$ in the NM and SSM, (e) Response of $i_{\mathrm{cq}}^{c}$ in the NM and SSM, (f) Response of $i_{\mathrm{cd}}$ and $i_{\mathrm{cd}}^{c}$ in the SSM, (g) Response of $i_{\mathrm{cq}}$ and $i_{\mathrm{cq}}^{c}$ in the SSM.

VSC are observed even under small phase jumps. Moreover, the $d q$-frame variables in the SSM show large mismatches from the NM, when system frequency deviates. Under events $i i$ of system frequency deviation, the small-signal stability analysis of VSC should be evaluated at a steady-state frequency.

\section{REFERENCES}

[1] L. Harnefors, M. Bongiorno, and S. Lundberg, "Input-admittance calculation and shaping for controlled voltage-source converters," IEEE Trans. Ind. Electron., vol. 54, no. 6, pp. 3323-3334, Dec. 2007.

[2] H. Zhang, L. Harnefors, X. Wang, J. P. Hasler, S. Östlund, C. Danielsson, and H. Gong, "Loop-at-a-time stability analysis for grid-connected voltage-source converters," IEEE Trans. Emerg. Sel. Topics Power Electron., pp. 1-1, 2020.

[3] P. S. Wright, P. N. Davis, K. Johnstone, G. Rietveld, and A. J. Roscoe, "Field measurement of frequency and ROCOF in the presence of phase steps," IEEE Trans. Instrum. Meas., vol. 68, no. 6, pp. 1688-1695, 2019.

[4] A. Ulbig, T. S. Borsche, and G. Andersson, "Impact of low rotational inertia on power system stability and operation," IFAC Proceedings Volumes, vol. 47, no. 3, pp. 7290 - 7297, 2014, 19th IFAC World Congress.

[5] Z. Zou and M. Liserre, "Modeling phase-locked loop-based synchronization in grid-interfaced converters," IEEE Trans. Energy Convers., vol. 35, no. 1, pp. 394-404, 2020.

[6] P. Kundur, Power System Stability And Control, ser. EPRI power system engineering series. McGraw-Hill, 1994. 